L'HOMME L'Homme

Revue française d'anthropologie

$190 \mid 2009$

Varia

\title{
Monnaie et dettes de vie
}

\section{Bruno Théret}

\section{OpenEdition}

Journals

Édition électronique

URL : http://journals.openedition.org/lhomme/22146

DOI : 10.4000//homme.22146

ISSN : 1953-8103

\section{Éditeur}

Éditions de l'EHESS

\section{Édition imprimée}

Date de publication : 1 janvier 2009

Pagination : 153-179

ISSN : 0439-4216

\section{Référence électronique}

Bruno Théret, « Monnaie et dettes de vie », L'Homme [En ligne], 190 | 2009, mis en ligne le 01 janvier 2011, consulté le 03 mai 2019. URL : http://journals.openedition.org/lhomme/22146 ; DOI : 10.4000/ Ihomme.22146

(c) École des hautes études en sciences sociales 


\title{
Monnaie et dettes de vie
}

\author{
Bruno Théret
}

$\mathrm{P}$

OUR FORMULER UNE THÉORIE de la monnaie susceptible de valoir pour un vaste ensemble de sociétés, et non pour les seules sociétés capitalistes actuelles, le livre La Monnaie souveraine, publié en 1998 et rassemblant des signatures d'anthropologues, d'économistes, d'historiens et d'un psychologue, posait comme point de départ d'une réflexion renouvelée sur la monnaie que celle-ci devait être rapportée au concept de dette plutôt qu'à celui d'échange. Toute société dotée de monnaie peut en effet être considérée comme un tissu de dettes/créances qui, par-delà leurs natures ou origines diverses, sont engendrées par des transferts de possession et/ou de propriété sur des biens réels ou symboliques. La monnaie est le médium qui donne une forme mesurable et quantifiée à cet ensemble de relations sociales constitutives de la société considérée ; par sa médiation, les interdépendances sociales qui prennent la forme d'obligations et de droits réciproques entre les sociétaires et entre ceux-ci et les organisations collectives représentatives du tout de la société sont traduites en termes de dettes et de créances.

La monnaie dans sa dimension d'unité de compte est dans cette perspective une représentation symbolique unitaire potentielle de la totalité sociale. Mais comme elle est également ce qui permet de faire circuler dettes et créances entre les membres de la société, elle donne aussi à cette totalité un caractère dynamique. En circulant dans la chaîne des paiements, elle est ce qui permet que les dettes soient honorées et donc relancées en permanence dans un cycle qui constitue le cœur de la reproduction sociale. En tant qu'elle est à la fois unité de compte et

À propos de Michel Aglietta \& André Orléan, eds, La Monnaie souveraine, Paris, Odile Jacob, 1998. Contributeur de l'ouvrage, l'auteur tient à remercier, d'une part, Jean-Michel Servet pour ses commentaires, d'autre part, Stéphane Breton qui, à travers un échange épistolaire serré de nos points de vue, lui a permis de développer et affiner ses arguments. Il reste néanmoins entièrement responsable des idées ici défendues. 
moyen de paiement, la monnaie est donc une médiation sociale clé de la formation et de la reproduction des sociétés. Elle les représente symboliquement en tant que tout, s'agissant là d'une représentation active qui participe à la construction et à la reproduction de ces touts qui ne lui préexistent pas nécessairement.

Dans La Monnaie souveraine, il est par ailleurs considéré que les dettes/créances ont leurs sources dans des transactions diverses: dons entre humains et entre humains et divinités ou autres esprits, échanges de type marchand, ou encore prélèvements centralisés et redistribués. Toutefois, pour saisir l'origine de la monnaie, il y est fait une place particulière, non pas aux échanges marchands, comme c'est le cas lorsque celle-ci est pensée à partir de la fable du troc, mais à la forme originaire ou primordiale qu' est la dette de vie. Cette forme de dette a sa source dans le fait que, dans toute société, les humains naissent, engendrent et meurent : ils reçoivent, donnent et rendent la vie. La vie fait donc l'objet d'une forme de don qui est à l'origine de relations d'endettement spécifiques, qu'on peut qualifier de dettes de vie : chaque être humain se voit en effet reconnu socialement doté d'un capital de vie ("réserve de vie ») plus ou moins important et plus ou moins valorisé selon les statuts sociaux, pouvant être approprié de diverses façons et faire l'objet de diverses transactions entraînant la création et la circulation de dettes. Ces dettes de vie sont au principe de la reproduction sociale car tout groupe, toute société est confrontée à la mortalité de ses membres, et pour s'inscrire dans la durée, doit faire face à la nécessité d'assurer sa pérennité par-delà leur disparition inéluctable à terme. Cette pérennité passe par la transmission des dettes de vie entre générations et l'entretien du " capital-vie " global de la société ${ }^{1}$, lesquels sont assurés par l'enchaînement, tout au long des cycles de vie individuels, d'un ensemble de transactions réelles et symboliques ritualisées liant les humains entre eux et aux êtres supra-humains - ancêtres, divinités, esprits, nation, patrie (à l'occasion des naissances, initiations, mariages, funérailles, meurtres, sacrifices, etc. $)^{2}$. La monnaie trouve dans cette perspective théorique son origine dans les paiements sacrificiels - comme substitut aux victimes vivantes -, mais aussi dans les paiements de compensation de déficits de capital de vie entre groupes créés par les échanges de femmes ou

1. Sur la notion de "capital-vie », cf. Hénaff (2002: 298-299) : «Tout se passe comme si, dans les sociétés traditionnelles (et à bien des égards cette représentation survit à leur disparition), existait un modèle de réserve de vie, un stock énergétique qui ne peut être entamé ou menacé sans que soit ressentie la nécessité de le restaurer [...]. Ce capital-vie, ensemble de personnes et de biens, de forces et de valeurs, de croyances et de rites, qui fondent l'unité et la cohésion du groupe, est figuré par deux symboles : le sang, symbole d'union et de continuité de la lignée et des générations, l'honneur, symbole de l'identité et de la différence qui permet à la fois de reconnaitre l'autre et d'exiger qu'il vous respecte». La vie comme fait biologique et comme existence sociale doit être maintenue " dans son intégrité ; toute atteinte appelle une action en retour, une procédure de compensation. Telle est la dette de vie» (id.). Cf. également Rospabé (1995).

2. Sur le rituel comme "source de vie " et ce qui «maintient l'unité du groupe et soutient la structure sociale ", cf. Lucien Scubla (1985 : 26-29) qui suit là Arthur M. Hocart (1978). 
les meurtres, et dans les paiements fiscaux qui apparaissent avec l'émergence de pouvoirs politiques centralisés assis sur un Trésor monétisable, tous paiements relatifs à différentes formes de dette de vie ${ }^{3}$.

Ainsi il est écrit dans La Monnaie souveraine que la dette primordiale :

" [...] est à la fois constitutive de l'être des individus vivants et de la pérennité de la société dans son ensemble. C'est une dette de vie. Dans son acception archaïque, cette dette est reconnaissance d'une dépendance des vivants à l'égard des puissances souveraines, dieux et ancêtres, qui leur ont consenti une part de la force cosmique dont elle est la source. Le don de cette force, qui permet à la vie de se maintenir, a pour contrepartie l'obligation des vivants de racheter, leur vie durant, cette puissance vitale dont ils ont été faits les dépositaires. Mais la série continuelle des rachats n'épuise jamais la dette originaire : elle construit la souveraineté et cimente la communauté dans ses travaux et ses jours, notamment à travers les sacrifices, les rituels et les offrandes [...]. L'hypothèse de la dette de vie rappelle que la société est menacée dans sa cohésion, voire dans son existence même, si elle n'assure pas les conditions de sa reproduction» (pp. 21-22).

Cette hypothèse faisant procéder la monnaie de la dette de vie a fait l'objet d'une série de critiques réunies, pour la plupart, dans le numéro Questions de monnaie, de L'Homme, publié en 2002 sous la direction de Stéphane Breton. Trois types très différents, voire opposés, de critiques peuvent être distingués.

Pour certains, dont l'historien Sylvain Piron, il faudrait purement et simplement l'abandonner, non seulement parce qu'elle serait inutile, mais surtout parce qu'elle conduit à mettre dans le même sac un fatras de choses hétérogènes simplement rassemblées «au prix d'un artifice » et par un «abus de langage » dans ce qui ne serait qu' "une nouvelle fable» rabelaisienne, un remake de la Dette de Panurge (Piron 2002: 258-259).

Pour d'autres, dont le sociologue et économiste Alain Caillé, elle reproduirait en l'entretenant "une sorte de vulgate " «intenable » qui témoigne, outre d'une "certaine ignorance de la littérature spécialisée ", d'une "illusion interdisciplinaire " aboutissant à substituer la dette de vie au don, le véritable invariant socioanthropologique dont il faudrait partir (Caillé 2002 : 246-249).

Pour d'autres encore, dont l'anthropologue Stéphane Breton enfin, elle serait au contraire parfaitement tenable et digne d'intérêt, mais aurait été paradoxalement poussée à la fois insuffisamment et trop loin ; d'un côté, elle oublierait une des modalités fondamentales de la dette, au cœur des sociétés exotiques, celle « relative à la reproduction sociale et à la règle exogamique de l'échange matrimonial» (Breton 2000 : 1364); de l'autre, elle postulerait que la monnaie procède de

3. Selon Lucien Scubla, il faut créditer Bernhard Laum d'avoir solidement établi «l'origine religieuse et même, plus précisément, sacrificielle de la monnaie " $(1985: 213$, n. 73) et «l'association de la monnaie non seulement à la procréation, mais aussi au meurtre et à la mort (même si c'est pour les opposer) peut difficilement passer pour contingente ...» (ibid., n. 69, 88). "Si on définit l'argent comme un moyen déterminé de paiement défini par sa nature et sa quantité, alors il faut considérer le culte comme la source originelle de l'argent " (Laum 1924: 158, traduit par Alban Bensa, "Présentation de Genèse et nature de la monnaie, de Bernhard Laum ", Genèses, 1992, 8 : 61). Cf. également de Coppet (1970) et Servet (1981). 
la dette en toutes circonstances alors que certains échanges sociaux, qui pourtant entraînent des formes de circulation monétaire, ne peuvent pas être assimilés à des rapports d'endettement (Breton 2002a : 20).

Cet article se propose de répondre successivement à ces trois espèces d'objection en cherchant à montrer qu'elles ne sont nullement dirimantes et qu'elles appellent plus un approfondissement de l'hypothèse que son rejet. Nos commentaires sont de deux types: on repousse d'une part certains arguments en leur opposant des contre-arguments non moins fondés; on mobilise d'autre part les critiques pour affiner et préciser le modèle retenu afin de renforcer les fondements de sa validité.

\section{Non à la dette!}

Le critique le plus négatif, le plus absolu, de l'idée de faire procéder la monnaie de la dette, et donc de sa forme première la dette de vie, est Sylvain Piron. Celui-ci doute que les «différentes significations de l'idée de dette, que le sens commun conçoit comme fortement hétérogènes, puissent à bon droit entrer dans la composition d'une même et unique figure structurale»:

«La confusion que l'on peut soupçonner dans cette mise en série concerne autant les ordres de phénomènes pris en compte que les niveaux d'analyse [...]. Ce n'est qu'au prix d'un artifice que l'on peut fondre au sein d'un seul concept une dette ontologique, constitutive de l'être social, avec les relations empiriques d'endettement qui n'expriment que l'une des modalités des rapports sociaux» (2002:258).

Comme en témoigne en outre son rejet, au nom d'une irréductible historicité des faits sociaux, de tout concept abstrait de monnaie susceptible de voyager (presque) partout, cet historien a une véritable difficulté avec la méthode comparative et la mise en relation de choses hétérogènes et a priori incomparables ${ }^{4}$. On peut en être surpris de la part d'un spécialiste de la monnaie, celle-ci étant précisément un " artifice » qui a pour vocation de mettre en relation de telles choses: des hommes et des femmes dans l'échange matrimonial ; une automobile, une baguette de pain et du savoir d'historien dans l'échange marchand ; des humains et des dieux dans les sacrifices; des peuples ennemis dans les tributs, etc. Dans La Monnaie souveraine, les dettes d'origines variées qui sont rassemblées dans le concept de dette sont dans cette perspective des dettes qui ont en commun d'entraîner des prestations monétaires, c'est-à-dire se traduisent par des paiements en monnaies; par là, elles sont quantifiées et tarifées, opération qui réduit leurs différences qualitatives - pourtant irréductibles - en différences quantitatives que l'abstraction de la numération permet alors de ramener à une commune mesure et donc d'agréger (ou de compenser). Il suffit de regarder un billet d'un dollar pour voir que la monnaie elle-même n'a pas la frilosité théorique de Sylvain Piron. N'affiche-t-il pas que This note is legal tender for all debts, public and private?

4. Sur le caractère assez général de cette difficulté chez les historiens, cf. Détienne (2000). 
En fait pour Sylvain Piron, rejoint sur ce point par Alain Caillé, les auteurs de La Monnaie souveraine seraient pris dans une «illusion transdisciplinaire" car cette «idée de dette pourrait avoir trop bien servi leurs intérêts convergents en leur offrant une figure anthropologique susceptible d'être convertie sans difficulté en un concept économique " (Piron 2002 : 258). Pourtant il concède que :

«[...] l'ossature de la définition de la dette comme lien social [...] est une ossature particulièrement robuste, et dont la validité universelle serait difficilement contestable. Sous le nom de "dette primordiale" est en effet visé, en un premier sens, le principe même de la primauté du social sur les éléments dont il se compose, la dépendance des individus envers la collectivité dans laquelle ils prennent place. Vérité élémentaire du fait social, évidence fondatrice de la possibilité d'une connaissance sociologique, la société est première, et c'est en son sein que naissent et se construisent les humains [...]. Mais ce trait à lui seul caractéristique de tout groupe humain, est d'une généralité telle qu'il ne dicte aucun mode particulier de formation des rapports sociaux. Donner à cette dépendance le nom de "dette" pour en faire le point d'ancrage structural de la circulation des dettes monétaires constitue donc soit un abus de langage, soit un raccourci saisissant qui demande à être développé " (Ibid. : 259).

Mais à quoi bon tenter de développer ce " raccourci », puisque Sylvain Piron préfere s'en tenir a priori à l'abus de langage. À peine a-t-il ouvert la porte à la possibilité de se laisser convaincre qu'il la referme par un jugement dernier : " cette appellation [...] égare davantage qu'elle n'éclaire» (id.), «l'emploi du terme est davantage porteur de confusion que de clarification » et «il est patent que celleci [l'institution monétaire] peut être parfaitement cernée sans qu'il y ait lieu de prononcer le mot de dette" (Ibid.: 261). Peut-être! C'est d'ailleurs ce que pensent la majorité des économistes et des sociologues parlant de monnaie, mais parlent-ils vraiment alors de l'institution monétaire? En effet, comme le reconnaissent des économistes aussi marqués par l'économisme que Carl Menger et Ludwig von Mises, il est difficile de parler d'un point de vue institutionnel (et donc notamment juridique) de la monnaie, sans parler de moyens de paiement et d'obligation de payer, c'est-à-dire de régler sa dette, ce qui revient implicitement à parler de dette. Dès lors qu'il s'agit d'étudier une monnaie située, historiquement et géographiquement, il devient nécessaire d'expliciter les types et formes de liens d'endettement qui prédominent dans le contexte analysé ${ }^{5}$.

À vrai dire, le rejet de la dette par Sylvain Piron est cohérent avec sa suggestion de rechercher une définition de la monnaie à partir d'une «analyse fondée sur les propriétés formelles des systèmes et des objets monétaires ", sa perspective étant même, afin de faire abstraction de l'abstraction monétaire qui ne serait caractéristique que de la monnaie moderne, "de faire porter l'accent sur les objets davantage que sur le système qu'ils forment " (Ibid.: 264). C'est un choix

5. L'échange marchand lui-même est créateur de dette, par-delà sa dissimulation par le paiement comptant : l'obligation de performance (livrer la marchandise) est doublée d'une dette de paiement (pour le transfert des droits de propriété sur les marchandises livrées ou à livrer). Sur ce point, cf. Commons (1990 [1934]) et Théret (2001). 
heuristique dont on attendra avec intérêt les résultats théoriques. En tout état de cause, il ne paraît pas nécessairement contradictoire avec celui fait dans $L a$ Monnaie souveraine qui conduit également à ne pas laisser à la théorie de la monnaie marchandise le monopole des choses monétaires, des "objets-monnaie ", de la «monnaie objectivée ${ }^{6}$.

En attendant, si on revient à la critique portée à l'idée de "dette en général ", homogénéisant indûment des obligations sociales d'origines diverses, deux réponses sont possibles. La première est apportée par Marcel Hénaff qui traite longuement de cette question. Ce dernier, malgré ses hésitations de départ tout à fait normales - «il n'est pas possible de manipuler sans de grandes précautions le concept de dette, ni de le faire sans indiquer à chaque fois le contexte de son emploi " (2002 : 272) -, conclut finalement d'une manière qui justifie la perspective ouverte par La Monnaie souveraine:

«Il n'en demeure pas moins que quelque chose de commun existe bien entre toutes les formes de l'obligation, et que se dessine un modèle sous-jacent dont l'insistance à travers les sociétés les plus différentes permet de supposer qu'il s'agit d'un invariant culturel majeur ; on pourrait le définir comme exigence homéostatique: la nécessité de rétablir un équilibre rompu " (id.).

L'autre réponse consiste à considérer qu'il n'y a pas de dettes purement financières ou purement symboliques ${ }^{7}$. Ce qui importe pour pouvoir parler de dettes, ce n'est pas leur origine, mais leur monétarisation. On peut ainsi poser qu'une dette non monétaire n'est pas une dette, mais une simple obligation (juridique ou éthique). L'abus de langage, au plan scientifique entendons-nous, serait, plutôt là, à parler de dette pour toute obligation sociale, même si celle-ci ne s'accompagne pas d'un paiement en monnaie; parler de dette pour une obligation sociale signifie qu'elle est évaluée par la médiation d'un système de compte (en unités de compte) et réglée par des moyens de paiements référés à ce système de compte.

\section{Le Don plutôt que la Dette!}

Alain Caillé ne partage pas le point de vue critique radical et d'obédience finalement assez orthodoxe de Sylvain Piron. Pour lui, partir de «l'affirmation d'une dette originelle» n'est pas entièrement faux, mais seulement dangereux du fait

6. Sur ce point, cf. Théret (2007). Ce souci de réintégrer les objets monétaires dans le cadre d'une analyse générale de la monnaie est également très présent dans les contributions des anthropologues au dossier Questions de monnaie, in L'Homme, 2002, 162.

7. «Le terme de dette ne doit pas être considéré comme appartenant d'abord au champ de l'échange marchand, ni même être défini prioritairement par rapport au contrat (le cas de l'Inde [...] suffirait à nous en convaincre). Il y a une dette symbolique plus large et plus profonde que la dette financière. Bien plus, celle-ci peut prendre aussi une dimension symbolique (perte de statut et état de dépendance du débiteur) [...]. Mais ce qui constitue le phénomène le plus intéressant, c'est le mouvement inverse; substitution à la dette symbolique d'une dette purement comptable. Une offense, un dommage ou même un meurtre involontaire peuvent être compensés par une somme d'argent [...] dont le montant est négocié entre le juge et les avocats des parties" (Hénaff $2002: 275$ ). 
que cela conduit à un holisme pur hypostasiant une Dette première "en surplomb». Alain Caillé préfere une autre hypostase, celle consistant à poser le Don sous sa forme maussienne, c'est-à-dire «l'obligation de donner, recevoir et rendre les présents» comme invariant anthropologique premier (2002: 250). Pour Alain Caillé, certes il n'y a "pas de don sans dette et sans circulation de dette" (ibid.: 251), mais la dette découle du don et ne saurait donc être le bon point de départ pour une théorie de la société.

Alain Caillé semble ainsi oublier que La Monnaie souveraine ne vise pas à fonder sur la dette une théorie de la société, mais tout au plus, éventuellement, une économie politique et, plus exactement, une théorie du fait monétaire destinée à en améliorer la compréhension. Ainsi limitée, l'hypothèse de la dette est moins aventureuse, plus acceptable a priori que celle alternative de son critique : elle ne présuppose pas que tout procède d'une seule forme de transaction dont toutes les autres découleraient - le Don maussien. La dette procède du don mais également d'autres types de transaction, qu'il s'agisse d'un don maussien, d'un "don indien" (Parry 1986), d'un don aux divinités, d'un échange marchand, d'un "échange identique", d'un tribut, d'un prélèvement avec redistribution, etc. Sauf évidemment à réduire tout type de transaction à une forme particulière de don, voie sur laquelle Alain Caillé semble, il est vrai, vouloir s'engager $^{8}$, sauf donc à tout ramener au Don, la dette est de ce fait un concept plus englobant qui vaut au-delà du don et pour tout lien d'obligation. Réciproquement, le don apparait comme un concept seulement second pour une théorie de la «monnaie en général » valant pour toute société ${ }^{9}$. Notons d'ailleurs que même dans la perspective d'Alain Caillé, on ne saurait fonder la monnaie directement à partir du don car on ne règle pas un don, on règle la dette créée par ce don, ce qui signifie qu'on est obligé de passer par le concept intermédiaire et unifiant de dette.

Cela dit, le point de vue d'Alain Caillé n'est pas nécessairement contradictoire avec les thèses de La Monnaie souveraine ${ }^{10}$. D'une part, en effet, il reprend à son compte l'idée que la monnaie procède de la dette de vie, pour le moins dans les sociétés sauvages; d'autre part, il définit le don comme un système d'obligations,

8. «Résumons donc notre propos de façon lapidaire. Loin d'être un produit dérivé de La Dette ou du Sacrifice, le don, en tant que système complet et complexe structuré sur la triple obligation de donner, recevoir et rendre, représente le véritable système de socialité originel dont les systèmes de La Dette ou du Sacrifice ne sont que des moments particuliers, plus ou moins autonomisés et hypostasiés à la suite d'évolutions historiques diverses. C'est en partant de ce système complexe, complet et premier du don qu'il faut tenter de comprendre la monnaie première, et non pas à partir d'un de ses moments dérivés, celui de La Dette et/ou du Sacrifice " (Caillé 2002 : 252). Cette idée d'une dérivation historique du système de La Dette et/ou du Sacrifice à partir du système premier du Don paraît contradictoire avec l'affirmation du même auteur par ailleurs que la "différence entre monnaies "sauvage" et "moderne" [est] proprement incommensurable" (Ibid.: 253).

9. Ce qui peut expliquer, dit en passant, que les théoriciens du don n'aient pas produit de théorie générale de la monnaie alors que La Monnaie souveraine peut avancer en ce sens en partant de la dette. 10. Comme le remarque également Michel Aglietta dans sa contribution «Universalité et transformations de la monnaie : la nature des crises monétaires ", in Théret, ed. (2007 : II, 17-43). 
ce qui implique que si ces obligations sont monétisées, alors le don est dette, suivant la définition de la dette comme obligation monétisée ${ }^{11}$. Ainsi peut-on dire que La Monnaie souveraine définit la dette de vie comme don initial de la vie dès lors que ce don crée une dette de paiement.

Toutefois, ces dernières considérations n'empêchent pas que le torchon brûle, car la prise de position d'Alain Caillé correspond à une divergence plus profonde qu'on peut schématiser en une formule quelque peu absconse : l'horizontal procède-t-il du vertical ou, à l'inverse, le vertical de l'horizontal ? Lequel est premier ? En effet, en privilégiant la dette de vie, qui plus est une dette de vie saisie d'abord comme le fruit d'une relation entre humains et puissances souveraines, La Monnaie souveraine est verticaliste, alors qu'en privilégiant le Don maussien, Alain Caillé est horizontaliste. On retrouve ici un débat récurrent en anthropologie qu'il n'est pas dans notre objectif, et d'ailleurs de notre compétence, de trancher. Il nous suffit de montrer que l'hypothèse d'Alain Caillé, en dépit de ce qu'il affirme, n'est pas mieux établie et donc plus légitime au plan scientifique que celle de La Monnaie souveraine. Elle est, selon nous, au contraire plus fragile, ce qui justifie qu'on maintienne l'hypothèse effectivement verticaliste de la dette de vie ${ }^{12}$.

Pour le montrer, on développera trois contre-arguments fondés sur la contestation du primat exclusif qu'à partir d'une lecture purement lévi-straussienne de Mauss, Alain Caillé accorde à la réciprocité dans les échanges. Nous les empruntons principalement au travail déjà ancien de Lucien Scubla (1985) sur les logiques de la réciprocité. Le premier consiste à montrer que certains arguments utilisés par Alain Caillé pour justifier son point de vue "contractualiste » ne jouent pas forcément en sa faveur. Le second conteste l'interprétation de la dette de vie qu'Alain Caillé emprunte à Philippe Rospabé (1995), interprétation qui veut que les paiements (verticaux) sacrificiels soient dérivés (comme les paiements cérémoniels) des paiements (horizontaux) de type bridewealth dans les échanges de femmes et de type wergeld pour la compensation des meurtres. Le troisième rejette l'interprétation évolutionniste que donne Alain Caillé de ce qu'il appelle le don diagonal ou transcendant (dons aux dieux, sacrifices) comme étant l'expression des seules grandes religions universalistes.

Alain Caillé considère que privilégier le don, c'est partir de l'obligation de donner, alors que partir de la dette revient à privilégier «l'obligation de rendre pour en faire la vérité de l'ensemble». Dit autrement, il faudrait privilégier le

11. "On objectera peut-être qu'il n'y a aucun avantage à mettre l'accent sur la dette plutôt que sur le don et d'autre part que si l'obligation d'être en dette est mutuelle et universelle, on retombe sur l'obligation d'échanger. Ce à quoi on répondra que la dette est première dès lors qu'il y a obligation ou devoir (de donner, d'échanger, de recevoir, ou de rendre) ; car dette ou devoir, c'est tout un» (Scubla 1985: 82).

12. Ce qui ne signifie pas que l'hypothèse horizontaliste soit nécessairement intenable, mais alors sa valeur devrait être testée par sa capacité à produire une théorie de la monnaie plus pertinente que celle tirée de l'hypothèse verticaliste. Or, jusqu'à maintenant, l'hypothèse horizontaliste a été principalement explorée par la théorie économique standard dont il est largement reconnu, y compris par Alain Caillé, qu'elle bute précisément et irrémédiablement sur une incapacité à intégrer le fait monétaire dans toute sa complexité sociologique. Cf. sur ce point les travaux d'André Orléan. 
point de vue du créancier sur celui du débiteur. Mais cela change-t-il grand chose d'un point de vue relationnel pour lequel il n'y a pas de débiteur sans créancier, d'autant plus que dans le don maussien, rendre c'est donner à son tour ? La relation est circulaire: puisque dans les échanges de dons, "aucun paiement n'abolit jamais aucune dette et [...] [que] par conséquent tout le monde est toujours endetté" (Alain Caillé in Rospabé 1995: 18), un débiteur n'est jamais qu'un futur créancier ${ }^{13}$. En outre, les clans entre lesquels s'échangent les épouses sont souvent simultanément débiteurs et créanciers. En quoi alors privilégier l'obligation de rendre plutôt que celle de donner biaise-t-il la vérité de l'ensemble?

En fait, on a l'impression qu'Alain Caillé voit dans le don réciprocitaire une relation qui n'est pas véritablement de dépendance et n'engendre donc pas une " vraie » dette, supposée fondamentalement verticale, à l'instar de Marcel Hénaff qui distingue quant à lui la «dette de réplique» - qui «naît et s'annule dans la rotation des échanges" - de la "dette de dépendance » - qui a tendance "à s’accumuler au profit des uns et au détriment des autres" $(2002: 278)^{14}$. Certes Alain Caillé reconnaît qu' «assurément dans l'obligation de donner, il entre, constitutivement, de la dette. Ou, pour mieux dire, la triple obligation maussienne est indissociable de l'obligation de s'endetter et de s'acquitter de ses dettes sans jamais pour autant sortir de l'état de dette" (Caillé 2002 : 251). Mais alors comment une relation d'endettement dont on ne peut pas sortir peut-elle être qualifiée d'horizontale ou contractuelle?

Ainsi, dans les échanges matrimoniaux, la relation peut certes apparaître comme « interactionniste, purement horizontale », mais sa "vérité d'ensemble » est une dépendance verticale vis-à-vis de la totalité sociale qui renvoie à un principe de reproduction sociale, à l'obligation sociale de respecter le tabou de l'inceste et la règle d'exogamie ${ }^{15}$. C'est là l'exemple même de la nécessité de « remonter en amont de la réciprocité » pour rechercher «l'ancrage nécessaire du

13. «Le donneur d'hier est le receveur d'aujourd'hui» (Hénaff $2002: 276$ ).

14. Il est intéressant de noter que Marcel Hénaff, pris dans le vocabulaire de sens commun en philosophie, se croit obligé d'ajouter que c'est seulement dans le second cas qu'il «s'agit rigoureusement de dette" alors que les dettes qui tournent, dont on peut se libérer, ne seraient pas des dettes «au sens strict, ou du moins au sens fort " (id.). Mais la rigueur dont il est question ici n'est que celle dictée par une préconception de la dette qui l'assimile a priori à une inégalité de positions et à une dépendance (dont on ne peut se libérer). Elle n’est pas celle du sens commun des économistes pour qui, à l'inverse, la doxa veut que les "vraies" dettes soient les dettes contractuelles, négociables et transférables, les dettes de vie dont on ne peut pas se libérer, les authoritative debts, n'étant pas des dettes, mais des dons, des dots, des impôts, des cotisations sociales, etc. Même un économiste aussi averti de cette question, l'institutionnaliste américain John Commons (1990), après avoir introduit la distinction entre authoritative debts et authorized debts (les dettes privées contractuelles) se sent tenu, pour se conformer au langage courant, de ne conserver le terme de dette que pour ces dernières, à l'inverse donc de Marcel Hénaff.

15. Alain Caillé n'évoque pas la règle exogamique et présente les échanges matrimoniaux comme de pures relations pacificatrices entre groupes égaux et rivaux; on est alors dans le cas d'une « dette interactionniste, purement horizontale, qui circule entre des groupes, des clans, des lignages de puissance approximativement égale, et justement pour exprimer leur parité $[\ldots]$. .../... 
plan horizontal sur le plan vertical» (Scubla 1985 : 37). Il est en effet impossible "de décrire complètement les échanges à l'aide de la seule dimension horizontale» et sans tenir compte des «rapports de la réciprocité et de la hiérarchie et (de) la subordination de l'axe horizontal à l'axe vertical» (Ibid.: 44). C'est dans cette nécessité qu'est fondée l'idée développée dans La Monnaie souveraine selon laquelle les échanges dans l'ordre marchand, d'apparence purement horizontale, mobilisent pour se reproduire la verticalité d'une monnaie souveraine, représentation du tout social.

Alain Caillé propose en fait une lecture de Mauss démesurément tirée, à la manière de Lévi-Strauss, vers la réciprocité. La Monnaie souveraine paraît quant à elle plus proche de la position originelle de Mauss si on suit l'analyse suivante de Lucien Scubla :

"Des trois obligations qu'il distingue (celle de donner, celle de recevoir, celle de rendre), [...] Mauss étudie principalement la dernière; mais il est le premier à le dire : le devoir de rendre implique le devoir de donner et celui de recevoir. Or, puisque rendre est un devoir, qu'est-ce que le devoir de recevoir, sinon le devoir d'avoir toujours à rendre et donc, comme le devoir de donner, le devoir d'être toujours en dette. Comme si l' "Essai sur le don" était aussi l'esquisse d'une théorie de la dette primitive " (Ibid. : 24-25).

Cela dit, la primauté du don sur la dette qu'Alain Caillé met en avant ne l'empêche pas d'être à nouveau proche de la thèse de La Monnaie souveraine concernant la relation entre monnaie et dette de vie. Avec Philippe Rospabé (1995), il admet en effet un "couplage étroit des monnaies sauvages avec la dette de vie " (Caillé 2002: 251). Pour lui :

" [...] la signification première de la monnaie [...] est reconnaissance du don d'une vie [...]. Cette identité de la monnaie et de la vie permet d'éclairer simplement mais de manière étonnement précise la signification d'autres pratiques dans lesquelles interviennent également des versements de monnaie : le paiement du wergeld, le don cérémoniel, et le sacrifice. Dans chacun de ces cas en effet, on est renvoyé au même signifié ultime, la dette de vie, mais de plus en plus médiatisé et symbolisé [...]. Dans l'institution du sacrifice enfin, on voit comment la reconnaissance de la dette de vie qui préside à la manipulation symbolique des biens précieux est adressée non pas à des donneurs vivants de la vie, pères ou frères, mais à des donneurs plus originels et insaisissables, les morts, les parents décédés" (Caillé in Rospabé 1995: 17).

[Suite de la note 15] La base de tout le système, [...] c'est la compensation matrimoniale, l'engagement pris de donner à son tour une épouse donneuse de vie en échange de celle qu'on prend. Chacun des groupes protagonistes du jeu cherche avant tout à maximiser le capital de vie dont il dispose et qui le constitue. Voici la vraie dette de vie première, celle que l'on souscrit en prenant épouse chez l'ennemi d'hier pour en faire l'ami de demain. Mais cette dette est purement horizontale» (2002: 251-252). Étrange formulation pour un anti-utilitariste convaincu. Étrange conception qui privilégie la nécessité de contracter une alliance pacificatrice avec l'extérieur en passant sous silence l'intériorisation de l'interdit interne d'endogamie qui conditionne la totalisation sociale. 
Ce par quoi Alain Caillé s'éloigne derechef de La Monnaie souveraine, c'est, toujours en suivant Philippe Rospabé, par la conception qu'il retient de la forme première de cette dette de vie : dette verticale à l'égard des puissances cosmiques dans La Monnaie souveraine, dette horizontale interactionniste entre groupes rivaux chez Philippe Rospabé et Alain Caillé pour qui le rôle de la monnaie dans les paiements sacrificiels ne fait que dériver de son rôle premier de "paiement pour la fiancée " dans les échanges horizontaux de femmes. Dans cette perspective, il distingue trois systèmes de don:

- un système du don horizontal « qui permet aux guerriers de passer de la guerre à l'alliance grâce au don des paroles, des biens précieux et des femmes ";

- un système du don vertical, "celui du don de la vie et de la fécondité " à travers lequel «se joue l’alliance entre les générations, la liaison du passé et de l'avenir (là où le don horizontal lie les contemporains)»;

- un système du don diagonal ou transcendant, « système du don aux esprits, aux entités supérieures et aux dieux" "dont l'autonomie ne va pas de soi " et "n'est nullement donné d'emblée» (Caillé 2002 : 250).

Alain Caillé soutient alors à l'encontre de La Monnaie souveraine que :

"Ce n'est que dans le cadre des grandes religions universalistes que le troisième système conquiert son indépendance et semble se subordonner les deux autres en interprétant les dons concrets entre les hommes (et les femmes) concrets, les dettes qui les lient et les offrandes aux forces spirituelles comme la projection d'une Dette première envers les dieux dont on ne saurait s'acquitter que par [...] le Sacrifice. Le religieux universaliste se forme ainsi en hypostasiant les principes qui sont à l'œuvre au sein du don horizontal et vertical. D'un point de vue se voulant scientifique, il est évident que c'est du don horizontal et vertical quil faut partir pour comprendre le don transcendant [c'est nous qui soulignons]. Au contraire poser la Dette, le Sacrifice, la Souveraineté comme préalables à la constitution des sociétés revient en définitive à tenter d'expliquer la société par la religion. Nous croyons pour notre part, au contraire, que l'hypostase historique réelle (les grandes religions existent bel et bien) des notions de dette, de sacrifice et de souveraineté doit être comprise comme le résultat d'une transformation du système du don premier (le couplage du don horizontal et du don vertical) qui possède sa cohérence propre et intrinsèque" (Ibid. : 250-251).

Cette double thèse - autosuffisance des dons de vie entre humains pour la construction et la reproduction sociale et engendrement évolutionnaire du don transcendant à partir de ces dons premiers dans le cadre des grandes religions universalistes - est évidemment contestable "d'un point de vue scientifique ", en dépit de ce qu'affirme péremptoirement son auteur. Elle est d'ailleurs contestée, notamment par Lucien Scubla qui soutient la thèse strictement opposée ${ }^{16}$. Pour ce dernier, le fait que le supra-humain, les croyances religieuses et le sacrifice

16. C'est également le cas de Maurice Godelier, "étonnamment proche» de Scubla comme l'a remarqué ailleurs Alain Caillé lui-même: "Godelier est conduit à subordonner étroitement les dons faits entre les hommes aux dons faits aux dieux, à prétendre déduire en somme les premiers des seconds, alors que seule la position inverse nous semble avoir du sens " (Caillé 2000 : 188). 
soient d'emblée présents dans les relations d'alliance matrimoniale et de filiation apparaît dans la structure même de l'atome de parenté mis à jour par Claude Lévi-Strauss où ils prennent la figure de l'oncle maternel (tiers exclus des démarches dualistes) et rendent compte de la relation avunculaire ${ }^{17}$. Selon cette interprétation, on serait là :

« [...] à la source de la première dette, sinon à celle du sacrifice ; [...] au lieu même où se conclut le premier contrat social [...]. Tout cela est inscrit dans l'atome de parenté ; si Lévi-Strauss lui-même manque à bien le voir c'est faute de reconnaître que les relations verticales sont le fondement des relations horizontales et que la réciprocité n'est pas une donnée immédiate mais le produit du croisement de relations asymétriques " (Scubla 1985: 60).

On est donc en présence de deux positions radicalement opposées, l'une, celle d'Alain Caillé, qui refuse de "tenter d'expliquer la société par la religion ", les croyances, l'autre, celle de Lucien Scubla, qui le juge nécessaire tout au moins en ce qui concerne sa genèse ${ }^{18}$. Il est clair que La Monnaie souveraine penche de ce second côté. Ce qui est cohérent avec le fait que la monnaie y est liée à la souveraineté, dont on a vu qu'elle était elle-même liée à la mort et à sa représentation ${ }^{19}$. Or cette question de la mort et de la souveraineté n'a pas droit de cité dans l'approche par le don à la Caillé-Rospabé qui ne se préoccupe guère, on l'a $\mathrm{vu}$, de l'origine des obligations de donner, recevoir et rendre. La monnaie y est bien associée à la vie, à la dette de vie, mais celle-ci n'est pas mise en relation avec la mort, si ce n'est secondairement dans le cas de la mort par meurtre (et des paiements pour le prix du sang). Pourtant, l'échange de femmes en vue de la reproduction du groupe suppose déjà là une représentation de la relation entre vie et mort ; elle suppose notamment qu'on ne croît pas à l'immortalité de l'homme,

17. Lucien Scubla établit en effet une équivalence entre "prix de la fiancée " et "prix de l'enfant " (prix pour le renoncement de l'oncle maternel à l'enfant de sa sœur), et oncle maternel et prêtre ou "dieu sanguinaire" (qui implique l'équivalence entre prix de l'enfant et victime sacrificielle) (1985: 57-60). On retrouve cette figure de l'oncle en position de dieu sanguinaire dans les mines de métal précieux des Andes sous les traits du Tio, esprit de la montagne, dispensateur de ses richesses minières, réclamant en retour des sacrifices pouvant aller jusqu'à la personne même du mineur. Cf. : Mike Sallnow in Parry \& Bloch, eds (1989: 213-214); Absi (2008).

18. Stéphane Breton soutient, quant à lui, qu’il y a, dans le cas des sociétés mélanésiennes, solution de continuité entre la logique économique horizontale des "compensations corporatives" (paiements pour un mariage et pour un meurtre) mettant en jeu des relations entre groupes et celle verticale de la filiation et de la "transmission" (paiements funéraires) liant les vivants au tout de la société représenté par les ancêtres. «Le don de monnaie n'est pas dans ce cas un paiement à proprement parler, puisqu'il n'éteint aucune dette et n'attend pas de contrepartie : il est pure dépense. [...] du point de vue mélanésien, c'est parce que la monnaie est un objet de don, marque de la transmission filiative ou de la communauté de relations à laquelle on donne le nom de société, qu'elle peut être un signe de la valeur dans l'échange " (Breton 2002a : 21-22). Il ne saurait y avoir, en ce cas également, dérivation des paiements sacrificiels de ceux concernant le prix de la fiancée et le prix du sang.

19. «La monnaie représente les morts ; c'est-à-dire que la clef de voûte des relations horizontales qu'entretiennent les vivants les uns avec les autres se trouve dans la relation verticale qui les rattache aux morts» (Scubla 1985: 38). 
car si on ne meurt pas, on n'a pas besoin de sexualité reproductrice et donc d'échange matrimonial (von Fürer-Haimendorf 1974: 540). Pour s'obliger à donner, recevoir et rendre la vie, il faut d'abord que la mort soit pensée. Ainsi une représentation de la mort est une pré-condition de l'échange des femmes. Cette représentation est première et joue en amont de cet échange.

Enfin, il est difficile d'accepter pour argent comptant l'idée que l'humanité a attendu l'émergence des grandes religions universalistes pour penser son rapport à la transcendance et se représenter comme endettée à l'égard de l'en deçà et de l'au-delà de la vie sur terre. L'hypothèse que reprend Marcel Hénaff de l'apparition d'une dette de dépendance envers les puissances cosmiques liée au fait que les humains empiètent sur leur domaine en cherchant à maîtriser la nature paraît plus réaliste ${ }^{20}$, moins religieuse, et elle va à l'encontre d'une telle conception. En effet, la représentation verticale de la dette de vie, dette de dépendance envers les dieux, corrélée à l'avènement du sacrifice et à l'apparition des sociétés de pasteurs et d'agriculteurs :

«[...] peut apparaître selon plusieurs formules [...] : 1) dette constitutive des Veda : le mode antérieur est effacé ; l'univers commence avec la dette parce qu'il est dit aussi commencer avec le sacrifice qui le fait être comme monde ordonné et le désigne comme objet d'une tâche à accomplir ; 2) dette événement de la tradition chrétienne [...] : le passage est représenté comme une chute, une faute due à l'orgueil des hommes; cela appelle une logique de rédemption ; 3) [...] dette cosmique conçue comme un déséquilibre entre les éléments, comme engendrée par le désaccord entre les hommes et les dieux ; cette dette n'est ni originaire comme un état, ni survenue comme une faute; elle est engendrement d'un écart à l'équilibre. Elle représente sans doute la forme la plus courante des cosmogonies et systèmes de pensée traversant des aires de civilisation agropastorales fort disparates: de l'Europe aux Amériques, de l'Afrique subsaharienne à l'Asie. Tel est le cas de la Grèce ancienne, certainement " (Hénaff 2002: 301) ${ }^{21}$.

Dans cette troisième forme, elle n'est nullement intrinsèquement liée à une grande religion universaliste. Il a également été remarqué que les sociétés qui pensent que certaines actions humaines, telles que la violation des tabous et des codes moraux, entraînent une intervention de pouvoirs surnaturels qui affectent

20. Selon Christoph von Fürer-Haimendorf (1974: 553-554), les sociétés qui n'établissent aucune relation causale entre les actions humaines de nature morale et l'intervention de pouvoirs surnaturels sur le sort des hommes sur terre et/ou après la mort sont principalement en effet des sociétés de chasseurs-cueilleurs, d'agriculteurs itinérants, ainsi que quelques communautés agricoles dotées de systèmes d'irrigation élaborés et des segments des sociétés industrielles modernes.

21. Ce dernier modèle "présente le monde comme un réservoir d'énergies dont l'usage est limité et défini : elles peuvent se déchaîner, déborder; il importe alors de savoir articuler le monde humain et le monde divin pour que ces excès soient maîtrisés (c'est, on l'a vu, une des fonctions majeures du sacrifice : rendre aux dieux ce qu'on leur a pris ; payer le supplément de vies produites par des vies immolées; maintenir la séparation des deux mondes et leur juste communication). Il y a dette chaque fois que cet équilibre est rompu. Alors tout malheur social (trouble politique, épidémie, échec devant l'ennemi) apparait comme le signe de cette rupture cosmique et suscite la nécessité d'offrir la compensation qu'il plaira aux dieux d'indiquer » (Hénaff 2002 : 301). 
le sort des hommes pendant et/ou après leur vie, incluent une grande diversité de sociétés, y compris des sociétés primitives sans écriture (von Fürer-Haimendorf 1974 : 554).

Cela dit, la distinction introduite par Alain Caillé entre "trois systèmes de don ", dès lors qu'on la dépouille de son aspect normatif faisant du troisième système une dérivation tardive des deux premiers, attire à juste titre l'attention sur une lacune du concept de dette de vie tel qu'il est présenté dans La Monnaie souveraine. Or c'est là une lacune qui a été également signalée par Stéphane Breton dans son analyse critique, que nous abordons maintenant, du statut de la dette dans l'ouvrage.

\section{La dette, oui mais...}

Comme nous l'avons noté en introduction, les critiques faites à La Monnaie souveraine par Stéphane Breton paraissent au premier abord paradoxales dans la mesure où celui-ci apprécie d'abord très positivement l'approche proposée par le livre, en suggérant seulement qu'elle soit complétée par une meilleure prise en considération de la dette de vie entre humains (celle qu'Alain Caillé place au centre de son analyse), puis adopte une position de retrait en mettant en avant l'idée que certaines relations monétaires ne sauraient être rapportées à une quelconque dette. Examinons successivement ces deux points, ce qui nous conduira à préciser le concept de dette ${ }^{22}$.

Contrairement à Sylvain Piron et même à Alain Caillé, Stéphane Breton est, comme l'historien Jean-Yves Grenier (2000), plutôt favorable aux thèses de $L a$ Monnaie souveraine. Il souligne la qualité du livre et «l'intérêt de sa contribution théorique ( Breton 2000 : 1366), notamment de son « déplacement conceptuel [qui] a tout lieu d'intéresser l'anthropologue qui se trouve bien souvent dans la position d'observer des usages monétaires échappant à la règle du marché et du principe d'équivalence" (Breton 2002a: 14). Sa "seule réserve» concerne la notion de dette, notamment parce qu'elle ne lui parait pas s'appliquer parfaitement au cas mélanésien. Cela pour deux raisons.

La première est qu'une modalité importante de la dette de vie «n'est pas l'objet d'une attention particulière de la part des contributeurs de ce livre", à savoir celle qui touche aux structures de parenté et qu'on peut qualifier de domestique. Pour Stéphane Breton :

« [...] le concept de dette employé [...] [dans La Monnaie souveraine] - équivalent au concept de lien social - recouvre non pas deux mais trois modalités. Les deux premières explicitement décrites par les auteurs, sont d'une part, la dette "verticale" entre les sujets et le tout social qui leur préexiste et les engendre - on peut parler d'une dette sociale ; d'autre part, la dette "horizontale" entre les sujets déterminés à échanger par la division

22. Outre les deux textes déjà cités de Stéphane Breton, publiés dans Annales. Histoire, Sciences Sociales (2000) et L'Homme (2002b), nous utilisons dans ce qui suit une correspondance personnelle avec lui grâce à laquelle nous avons pu affiner nos positions respectives. 
sociale du travail - on peut parler de dette privée. Mais celle-ci est indirectement sociale car la division du travail ne résulte pas d'un accord sur le marché. C'est à ce point qu'entre en jeu [...], la troisième modalité, celle de l'interdit symbolique, modalité relative à la reproduction sociale et à la règle exogamique de l'échange matrimonial, qui subsume les deux modalités précédentes puisqu'elle est à la fois le fondement de toute division du travail et l'expression la plus élémentaire de la dette privée, laquelle s'établit entre affins» (2000: 1364).

Cette critique est pour nous totalement justifiée ${ }^{23}$. Elle rejoint celle d'Alain Caillé concernant ce qui, dans La Monnaie souveraine, serait une hypostase de la dette de vie verticale requalifiée par lui de don diagonal. Il semble d'ailleurs judicieux de reprendre cette idée de diagonalité, mais pour qualifier non pas la dette vis-à-vis des puissances souveraines qui est bien, quant à elle, strictement verticale, mais celle inscrite dans les structures de parenté et engendrée par le tabou de l'inceste. Celle-ci combine en effet étroitement la dimension horizontale de l'alliance matrimoniale avec celle verticale de la filiation (la résultante d'un vecteur vertical et d'un vecteur horizontal est un vecteur diagonal).

En introduisant cette troisième modalité de la dette, on obtient une structure triadique et non plus dyadique, la dette "sociale " étant dédoublée en dette de vie entre entités humaines (personnes et/ou groupes) et dette de vie entre humains et puissances souveraines. Ces deux formes de dette de vie ont en commun d'être des dettes dont on ne peut pas se libérer sauf par la mort ou un autre geste souverain (exil volontaire ou encore révolution politique par exemple), ce en quoi elles s'opposent ensemble à la dette horizontale privée qui a une source contractuelle et dont on peut se libérer dans le cours de la vie humaine. Aussi peut-on considérer, en reprenant les catégories de Maurice Bloch et Jonathan Parry (Parry \& Bloch 1989), que les dettes verticale et diagonale sont constitutives de l'ordre de long terme des sociétés - elles participent toutes deux de leur reproduction généalogique -, tandis que les dettes horizontales interindividuelles appartiennent à l'ordre de court terme - elles ne participent qu'indirectement (à travers la médiation de la monnaie) à la reproduction sociale puisqu'elles sont régies par une logique individualiste. Il en découle que, outre le problème de l'articulation entre ordres de long et de court termes, il doit y avoir au sein même de l'ordre de long terme une certaine cohérence et hiérarchie entre les deux formes verticale et diagonale de la dette de vie, hiérarchie qui sera différente selon que la totalisation sociale passe prioritairement par les rapports d'échanges matrimoniaux et la filiation (cas mélanésiens), ou par les relations aux puissances supra-humaines représentées par des Églises ou des États (cas de l'Inde, de la Chine, de l'Europe, etc.).

23. Aussi avons-nous nous-mêmes développé ultérieurement une meilleure prise en compte de cette dette de vie domestique dans le système général des dettes, notamment en observant que le dédoublement-renversement des formes de la dette de vie propre au capitalisme démocratique opérait non seulement au niveau de la dette verticale entre humains et puissances souveraines, mais également au niveau du système de parenté où il introduit des tensions extrêmement fortes entre la logique horizontale de l'alliance matrimoniale et la logique verticale de la filiation. Cf. Théret (1999, 2003). 
Les trois grandes formes de dettes ainsi définies constituent autant d'ordres transactionnels ayant leurs logiques propres, mobilisant a priori leurs propres moyens de paiement, et qui ne forment une communauté de paiement que moyennant le jeu d'une «monnaie-alliance» (Théret 1998 : 270-274). Celle-ci - système de compte commun et régime de monnayage assurant une articulation par conversion des diverses espèces de moyens de paiement - doit être suffisamment stable pour que l'unicité du compte ne soit pas menacée.

Toutefois la prise en compte de la dette diagonale ne saurait suffire à contenter Stéphane Breton. Il a en effet une deuxième raison d'être réticent vis-à-vis de la notion de dette telle qu'elle est mobilisée dans La Monnaie souveraine. Selon lui, en effet, l'hypothèse d'une dette primordiale, dette de vie verticale, à l'origine de la monnaie n'aurait pas de portée universelle, car elle ne vaut pas pour la société Wodani, son terrain d'étude anthropologique, et même plus largement pour l'ensemble des sociétés mélanésiennes. Les Mélanésiens ne vivent pas, en effet, leur lien au tout social, personnifié symboliquement par leurs ancêtres et notamment par un ancêtre fondateur et primordial, comme une dette à leur égard :

«En Mélanésie les vivants ont une notion claire de ce qu’ils ont reçu des ancêtres, mais ceux-ci ne leur demandent pas de remboursement, et ceux-là n'expriment ce lien que sous la forme du don: il appartient à chaque génération de transmettre ce qu'elle possède et ce qu'elle a reçu. Ce n'est d'ailleurs pas décrit sous la forme d'une obligation (comme l'est par exemple l'exogamie), mais d'une évidence [...]. Ce lien est celui de la transmission. Transmission ne vaut pas dette. Or les Wodani connaissent bien la notion de dette et de crédit [...]. Si ce schéma très clair n'est pas utilisé pour décrire la relation entre les vivants et les morts, par exemple, c'est qu'il n'est pas adéquat» (Breton, correspondance personnelle) ${ }^{24}$.

En conséquence, «la notion de dette ne peut être un concept général simplement parce qu'elle n'est pas une représentation locale en Mélanésie». Certes il y a bien en Mélanésie :

24. Ou encore, selon une communication plus personnelle récente dans laquelle Stéphane Breton, selon ses propres termes, réduit sa position à sa plus simple expression : «La monnaie revêt en Mélanésie deux aspects. Le premier est la fonction d'échange, intelligible du point de vue de la théorie économique. La monnaie est bien l'instrument de la libération d'une dette, à condition toutefois que l'on ne transforme pas celle-ci en une Dette de vie métaphysique, comme c'est le cas dans l'Inde védique et des castes, où cette formulation trouve son origine. Le deuxième aspect est celui de la monnaie comme substance, et sous cet aspect elle ne relève pas de la perspective économique. Il s'agit de substance sociale, sorte de valeur absolue et d'instrument génésique transmissible. En Mélanésie il s'agit d'une substance corporelle, correspondant à ce que les membres d'un clan ou d'une société ont en commun dans leur corps, dans leur personne, par engendrement et génération, comme, dans certains cas, la substance paternelle, à savoir les os. La monnaie est en somme l'incarnation, le mot convient bien, du social, la choséification des relations sociales. C'est un des rares supports dans lequel sont réifiées en Mélanésie les relations sociales (les autres sont le produit des jardins, qui échappent à la vente et sont partagés, le cochon, les substances et humeurs physiologiques enfin, qui se transmettent et « appartiennent » aux paternels ou bien aux maternels (cf. mon chapitre "La matrice masculine» dans Qu'est-ce quiun corps ?, Paris, Flammarion-Musée du quai Branly, 2006). C'est ainsi qu'il faut comprendre les 'Are'are (Daniel de Coppet) lorsqu'ils disent que la monnaie de coquillage est la substance des ancêtres, les Iqwaye (Jadran Mimica, communication personnelle), lorsqu'ils disent que la monnaie est du sperme, les Daribi (Roy Wagner), .../... 
« [...] une relation de dépendance entre la totalité sociale et les individus qui l'habitent [...]. [Mais] elle consiste tout simplement dans l'obligation de ne pas agir autrement que ne l'ont fait les ancêtres, ou dans le caractère inconcevable de ne pas faire autrement» (Ibid.) $)^{25}$.

\section{Aussi, pour Stéphane Breton :}

«[...] plutôt que de dire - ce qui à mon sens est une réification - que la monnaie est l'expression d'une dette fondamentale, on peut se contenter d'exprimer l'idée [...] qu'elle est l'expression d'une confiance sociale, d'une foi partagée dans les valeurs, qui peut prendre des formes diverses, dont celle de la dette " (Ibid.).

En outre, selon lui, le concept de dette, s'il permet de penser l'économie des choses, caractéristique des sociétés modernes, ne peut en revanche "rendre compte de l'économie des personnes" (Breton 2002a: 18) propre aux sociétés exotiques $^{26}$. En effet :

« [...] dans l'économie des personnes la monnaie n'est pas tant l'instrument d'un paiement que celui d'un transfert, d'une circulation, d'un don qui a pour objet de produire des relations sociales au sein desquelles et grâce auxquelles sont construites des personnes vivant dans une société qui se reproduit" (Ibid.: 15).

Stéphane Breton, finalement, pense que «la dette est une forme de relation sociale fondée sur la réciprocité et donc sur l'accord implicite ou explicite des contractants. Cet accord fait partie du concept et de la pratique de la dette [...]. Il n'y a de dette que si l'on s'est entendu pour qu'il y ait dette. La dette comme

[Suite de la note 24] lorsqu'ils disent que la monnaie est un œuf, les Wodani enfin (Stéphane Breton), lorsqu'ils disent que la monnaie est une personne, une personne immortelle. Sous cet aspect de substance sociale, la monnaie de coquillage n'est pas nécessairement l'instrument de l'extinction de la dette. Elle est aussi l'expression de ce qui échappe à l'échange et qui, pour être chose commune, subit au contraire la loi du partage. Par exemple, chez les Wodani, au sein d'un clan, pas de compensation de mariage ou de meurtre. L'exogamie établit la séparation entre la sphère du partage et celle de l'échange. Ce n'est qu'un clan étranger qui peut compenser un autre clan au moyen de monnaies. D'un côté moyen de l'échange, de l'autre incarnation de la chose sociale, la monnaie a deux visages. En Mélanésie, il va de soi que c'est parce qu'elle représente la substance commune qu'elle est la valeur ultime, et qu'elle peut donc servir aux échanges. C'est en somme, comme dans la théorie grecque de la valeur décrite par Louis Gernet, son origine, sa provenance mystique, qui lui prête ce caractère de valeur d'échange. Ce n'est pas parce qu'elle peut servir aux échanges qu'elle a de la valeur, mais c'est parce qu'elle est une incarnation de la communauté sociale qu'elle a une valeur d'échange. La monnaie est donc bien une représentation de la totalité sociale, mais sous la figure de la communauté et non sous celle du débit».

25. Selon Stéphane Breton, "nulle dette à l'égard du géniteur originaire n'apparaît» (correspondance personnelle).

26. " [...] il semble que dans les sociétés exotiques [...], l'échange ait moins pour but d'établir la valeur des biens échangés que la position respective ou le rang (doit-on dire valeur ?) des personnes qui les échangent. On peut parler d'économie des personnes dans la mesure où il s'agit [...] de droits sur celles-ci (compensation matrimoniale), de définition et de transformation de leur identité (compensation funéraire, paiement de filiation, échange cérémoniel), et enfin de constitution de groupes en tant qu'entités corporatives (compensation d'homicide, hiérarchisation de l'accès aux monnaies)» (Breton 2002a: 15). 
le don ne sont pas des faits objectifs, mais des faits sociaux, intentionnels " (correspondance personnelle). Pour Stéphane Breton, la dette est donc «intentionnelle ", ce qui «ne veut pas dire "volontaire" ou "librement consentie", mais est liée au caractère normatif du concept de dette, la relation de dette impliquant un système d'actes et de droits" (id.). La difficulté qu'a Stéphane Breton avec l'idée de dette telle qu'utilisée dans La Monnaie souveraine vient de ce qu'elle " est un concept objectif voulant se faire passer pour un concept subjectif et indigène " (id.). Bref, s'il y a bien en Mélanésie une situation objective de dette primordiale où prévalent des obligations qui vont se traduire par des paiements monétaires, celles-ci ne sont néanmoins pas pensées comme dette par les indigènes ${ }^{27}$.

Comment répondre à cette critique de portée potentiellement dévastatrice ? N'oblige-t-elle pas, en effet, si on l'acceptait telle quelle, à abandonner la formulation première selon laquelle la monnaie procède de la dette, et même plus fondamentalement de la dette primordiale et verticale de vie à l'égard de ce qui représente l'immortalité ?

Pour cela, on commencera par contester la portée de l'argument qui voudrait que le concept de dette permette de penser "l'économie des choses " propre à la modernité occidentale, mais pas les sociétés exotiques où la dette ne pourrait totalement rendre compte de "l'économie des personnes». En effet, différencier les sociétés modernes des sociétés exotiques à partir de l'opposition entre économie des choses et économie des personnes n'est pas du tout dans l'esprit de $L a$ Monnaie souveraine, même si y est reconnue l'évidence que chacun de ces types d'économie domine respectivement dans ces deux types de société. Une idée importante portée par le livre est au contraire qu'il faut prendre en compte le fait, également évident, que l'économie des personnes n'a pas disparu dans les sociétés de marché généralisé et que, dans la reproduction sociale, elle y tient même une place cruciale quoique dominée ${ }^{28}$; c'est là une position symétrique à celle prise par l'anthropologie récente reconnaissant la place d'échanges de type marchand et de la logique individualiste dans les sociétés exotiques (Parry \& Bloch, eds 1989; Akin \& Robbins 1999). Un élément-clé du fonctionnement de la monnaie tant ici que là réside précisément dans l'articulation de ces deux types d'économie.

Il suffit de s'arrêter un instant sur le rapport salarial pour saisir combien l'économie capitaliste met une économie des hommes au fondement de son économie des choses. La «force de travail » que le salarié est présumé posséder et vendre sur le marché du travail n'est, en effet, qu'une objectivation symbolique de sa personne qui permet au travailleur de se soumettre au salarieur en échange d'argent tout en gardant le sentiment qu'il n’aliène pas sa liberté. Et en réalité, le salaire n’est pas

27. Stéphane Breton préfere parler de dépendance parce que ce n'est pas un concept normatif mais "objectif, et donc plus extensible, plus neutre, prêt à être spécifié, contrairement au concept de dette, qui est à la fois subjectif et réciproque..." (correspondance personnelle).

28. Comme cela ressort bien des expériences contemporaines de systèmes d'échanges locaux (SEL). Cf. Servet (1999); Blanc (2007); ainsi que les travaux sur le «marquage » des monnaies de Viviana Zelizer (2005). 
autre chose qu'un mode, empruntant une symbolique monétaire et juridique, de fixation de la valeur des hommes et non de cette chose que serait leur force de travail (ce qui impliquerait qu'elle soit véritablement détachable de leur corps). On risque ainsi de tomber dans le fétichisme de la marchandise dès lors qu'on prétend opposer économie des choses et économie des hommes. Le capitalisme salarial moderne est économie des choses certes, mais aussi économie des hommes, car, comme dans les sociétés exotiques, la fixation de la valeur relative des humains - y compris évaluée en monnaies - y est toujours en jeu. Celle-ci passe par le marché du travail, mais aussi concurremment par l'ensemble des institutions sociales qui reconnaissent à l'homme une valeur politique (droits associés à la citoyenneté et ouvrant éventuellement droit à des transferts monétaires tarifés) et une valeur culturelle (qualifications, compétences, créativité, etc.) susceptibles d'être converties, via le marché du travail, en valeur économique (Théret 1994a et b).

On peut par ailleurs souligner que Stéphane Breton, dans son analyse de la monnaie wodani, polarise son attention sur les seules compensations matrimoniales et pour meurtre, c'est-à-dire sur ce qu'on a requalifié de dette diagonale de vie ${ }^{29}$. Cela se comprend car la société wodani est égalitaire et sans pouvoir politique séparé. Mais cela a l'inconvénient d'exclure de l'analyse tant la sphère des échanges de type marchand (ordre de court terme des dettes horizontales dont on ne repère la présence que par l'équivalence existant entre monnaies wodani et rupiah indonésienne), que la sphère sacrificielle dont on subodore pourtant qu'elle n'est pas sans avoir une extension significative. Stéphane Breton souligne, en effet, que les notions de faute et de culpabilité existent bien chez les Wodani et qu'elles sont sanctionnées par des esprits méchants auxquels il faut offrir des sacrifices. Il observe notamment que :

«Les Wodani sont des mangeurs coupables. Ils se sentent d'ailleurs coupables en toutes choses, et les morts aux fantômes desquels ils sacrifient le plus souvent sont leurs frères aînés, rendus méchants par le spectacle du plaisir des survivants. Il ne faut pas oublier de leur sacrifier régulièrement un cochon" (Breton 2002b : 213).

Or «la consommation de nourriture est l'acte le plus problématique, et aussi le plus chargé de sens qu'ils connaissent. C'est l'acte social par excellence» (Ibid.). En effet, c'est l'acte par lequel s'opère la totalisation sociale, puisqu'on mange également (symboliquement) les monnaies. Manger dans son coin en cachette est une attitude individualiste répréhensible punie par les esprits : en d'autres termes, c'est de la fraude! Cela ne suggère-t-il pas que se plier à l'ordre établi n'est pas évident pour les Wodani, qu'il est concevable de ne pas le respecter, et que la «transmission" ne va pas réellement de soi ? ${ }^{30}$

29. Ce qui est une focalisation fonctionnellement équivalente à celle de l'économie mainstream réduisant la société moderne à son économie marchande et faisant abstraction de son économie fiscale et de son économie domestique.

30. On peut renforcer cet argument en rappelant également le rôle de Tege, esprit meurtrier qui veille à punir toute démesure, qu'elle concerne la culture de plusieurs jardins, ou la rétention des monnaies de grande valeur hors de la circulation monétaire (Breton 2002b : 212-213). 
Par ailleurs, Stéphane Breton considère que le déni de la dette verticale vaut pour l'ensemble de la Mélanésie. Or il semble que cette généralisation soit indue au vu du cas de la société de Mono-Alu décrite par Denis Monnerie (2002). Dans cette société, plusieurs mythes dont celui de Soi, « héros mythique et ancêtre originel » à qui on attribue " la découverte des cordons $[. .$.$] qui sont utilisés pour [\ldots]$ assembler les perles des monnaies les plus valorisées ", instituent une " coupure fondamentale $[\ldots]$ entre les ancêtres originels et le monde des vivants qui leur est sans cesse redevable" (ibid.: 88). "S'instaure ainsi entre les protagonistes du mythe comme une dette mythique inépuisable de la part des vivants. Les monnaies sont à la fois l'origine de cette dette et les biens qui visent à la combler " (ibid.: 90).

N'est-ce point là un cas où une dette de vie verticale est bien pensée comme dette, compensable en monnaie, des vivants à l'égard des ancêtres morts?

Pour réduire la portée de la critique de Stéphane Breton, on peut encore invoquer la raison qui fait qu'il adhère lui-même, malgré ses critiques, aux thèses de La Monnaie souveraine, à savoir sa reconnaissance du fait que la théorie économique standard n'est pas pertinente pour penser la monnaie dans la société moderne. En effet, dans la mesure où il considère par ailleurs, à juste titre selon nous, qu'elle n'est autre qu'une théorie indigène propre à nos sociétés, il reconnaît implicitement qu'une telle théorie ne dit pas nécessairement la vérité sur la société qu'elle théorise, ne traduit pas nécessairement ce que pensent unanimement l'ensemble de ses membres. Ainsi quand il observe qu' « on peut [...] étendre le terme de monnaie au cas de sociétés exotiques, sans se soucier de guillemets, si l'on précise que l'échange des utilités n'est qu'un cas particulier de la circulation de la valeur et que la théorie économique de la monnaie n'est qu'une théorie indigène, qu'il nous faut éclairer par comparaison avec d'autres théories indigènes" (Breton 2002a : 24), cela suggère que la théorie indigène des Wodani, quand elle nous assure que le don primordial de vie ne crée pas de dette mais doit seulement être réitéré31, que cette obligation relève de "l'évidence » ou $\mathrm{du}$ " caractère inconcevable de ne pas faire autrement ", ne dit pas nécessairement, elle aussi, la vérité de la société wodani.

Certes, Stéphane Breton a raison de considérer qu'il ne peut décrire le système social des Wodani "sans prendre en compte les raisons qu'ils se donnent et qui font partie de l'objet même" (correspondance personnelle). Mais "prendre en compte " implique-t-il de prendre pour argent comptant le point de vue ainsi exprimé ? Pour l'anthropologie, selon Stéphane Breton, oui ! Car parler de dette à propos de leurs relations aux êtres supra-humains reviendrait à penser que les Wodani «sont en dette sans le savoir », ce qui serait contraire à l'idée de dette telle qu'elle doit être conçue, c'est-à-dire comme concept subjectif et normatif (ibid.). Mais, si on adoptait la même attitude pour les sociétés capitalistes contemporaines où dans la théorie indigène - à savoir l'économie politique -, les seules dettes qui

31. «Pour les Wodani, si le monde connaît désormais l'échange, c'est parce que l'ancêtre primordial a commencé par faire un don - celui de la vie et de la chair. Cette dépense ne crée pas de dette, elle doit seulement être réitérée" (Breton 2002a : 24). Remarquons au passage que l'obligation de réitérer une dépense ressemble bougrement à une dette. 
sont pensées en tant que telles sont les dettes marchandes contractuelles, on ne devrait pas non plus parler de dette sociale pour ce type de société, comme le font La Monnaie souveraine et Stéphane Breton à sa suite. Aussi, il se pourrait bien que ce soit ce dernier et non le livre qui subisse "l'influence méconnue de l'économie $»^{32}$ et s'adonne au fétichisme de la monnaie sauvage dont parlent Maurice Bloch et Jonathan Parry et qui est associé à son anthropomorphisation.

Ce qui frappe dans la description que donne Stéphane Breton de la société wodani, c'est la stabilité de cette dernière, la reconnaissance non problématique des obligations qu'elle impose à ses membres, l'inconcevabilité qu'il puisse en aller autrement que de sacrifier à l'ancêtre primordial et aux fantômes des morts récents, tout cela témoignant d'une incorporation extrêmement forte de la contrainte de reproduction de la société et des clans ${ }^{33}$. Par ailleurs, paradoxalement, la société wodani apparaît très moderne d'un certain point de vue puisque, en quelque sorte, le rapport de souveraineté y est renversé comme dans les sociétés salariales démocratiques. C'est en effet l'ensemble des clans qui sont endettés vis-à-vis de leurs membres et non l'inverse :

"Contrairement à ce qui se produit dans le champ des dettes privées, où il revient au débiteur de s'acquitter de ses obligations, la créance matrimoniale à laquelle donne naissance la règle exogamique n’est pas nécessairement remboursable par le bénéficiaire luimême. Le plus souvent, ce sont les segments sociaux, clans ou lignées, sinon la société dans sa totalité qui se chargent d'affecter une épouse à celui qui avait ou non cédé une sœur. Les personnes ne sont pas débitrices en leur nom propre, et seule la société est créancière » (Breton 2000 : 1366).

La société wodani connaîtrait ainsi une sorte de protection sociale, de dette sociale, qui pourrait participer à la représentation que se font ses membres de leur relation au tout de la société et à l'ancêtre fondateur comme n'étant pas une relation de dette, mais de simple «transmission ». Ce qui suggère non pas de "reformuler la notion de dette" (Ibid.), mais de chercher ailleurs et autrement le rapport primordial d'endettement, la dette de vie verticale liant les clans, et non pas les individus, au tout de la société, de telle sorte que ces derniers ne se sentent pas directement endettés vis-à-vis d'elle. Un peu comme dans les sociétés salariales où la dépendance du salarié peut ne pas être ressentie comme telle, non seulement du fait qu'elle passe par un rapport marchand se présentant sous les formes de l'équivalence, mais aussi parce que la condition salariale est médiatisée par l'appartenance à des corps professionnels et autres caisses de sécurité sociale qui assurent à chaque salarié une protection sociale à l'égard de laquelle il se vit comme un créancier plutôt que comme un débiteur.

32. " [...] cette idée de Dette trahit encore, nous semble-t-il, l'influence méconnue de l'économie, qui pense que toute valeur trouve son fondement dans une équivalence, que le transfert des biens est borné par une attente de réciprocité, et que la dépense renvoie nécessairement à un gain d'utilité " (Breton 2002a : 17).

33. C'est là, semble-t-il, un trait caractéristique des sociétés mélanésiennes, qui a permis à plusieurs d'entre elles de résister à l'introduction par les colonisateurs de l'argent moderne en le soumettant à leurs « lois» (Akin \& Robbins 1999). 
Mais ce n'est là que spéculation osée. Seule l'analyse d'une situation de crise monétaire au sein de la société wodani ou de toute autre similaire pourrait nous éclairer sur ce qu'il en est véritablement. Comment la société wodani réagit-elle à l'introduction des rupiah indonésiennes par les jeunes hommes qui vont travailler à l'extérieur et reviennent munis de cash, désireux de le réinvestir dans les compensations matrimoniales? Est-ce que cela modifie les représentations de la «transmission"? Ces questions restent posées, mais le fait qu'on puisse se les poser montre déjà combien la problématique du dévoilement de la nature profonde de la monnaie par sa crise ${ }^{34}$, ne vaut pas que pour la monnaie moderne et son fétichisme particulier; elle vaut pour toute monnaie.

Venons-en alors à notre dernier contre-argument. Il concerne le caractère nécessairement réciproque de la dette pour Stéphane Breton ${ }^{35}$. Selon nous, au contraire, une dette n'implique nullement la réciprocité ou l'équivalence ${ }^{36}$. La dette de vie verticale n'est pas réciproque ; elle émane d'un don initial sans contrepartie, car ce don est incommensurable et ne saurait être retourné. Il appelle seulement des paiements qui n'ont nullement pour but de rembourser, de régler la dette, mais seulement de sanctionner le lien de dépendance inauguré par le don originel, de reconnaître qu'on est en dette, dette fondée donc sur la seule "foi sociale». Ces paiements, comme le don initial, sont donc des transferts sans contrepartie. L'impôt permanent en est la forme moderne. Dans le paiement de l'impôt, il n'y a pas de contrepartie monétaire si ce n'est celle, éventuelle et non nécessaire, d'une redistribution ultérieure sans rapport direct avec le montant de l'impôt ; la seule contrepartie est un devoir de protection, sans garantie d'exécution (autre que par la médiation du processus de légitimation de l'État), et le tarif fiscal n'est pas fixé en fonction de cette contrepartie (sauf pour les économistes libéraux qui, afin de réduire l'État à un simple producteur marchand, violent le réel en faisant comme si l'impôt était le prix des services que l'État fournit). L'impôt est en fait un paiement unilatéral qui témoigne d'une soumission à une souveraineté. Il ouvre certes le droit de bénéficier, dans certaines circonstances, de prestations sociales ou corporatives, mais celles-ci sont établies selon leur propre logique de protection, indépendamment de la contribution fiscale de chacun. Aucune logique d'équivalence et de libération du lien ne joue ici. C'est également clair dans le cas du salariat moderne : le paiement du salaire n'a pour contrepartie qu'une obligation, par ailleurs peu précise et aléatoire, de performance dans le travail, et il n'y a équivalence que si on la postule (Cartelier 2007).

34. Sur la dénaturalisation de la monnaie dans sa crise, cf. Carruthers \& Babb (1996) et Théret (2007).

35. «Il me semble que la dette est un concept applicable aux seuls faits où la réciprocité fonde la loi mécanique de l'échange, et que l'on court un risque à vouloir en faire une notion plus générale» (Breton $2000: 1366)$.

36. C'est parfois aussi l'opinion de Stéphane Breton qui, au détour d'une page, nous apprend que «si c'est à l'occasion de funérailles ou du sacrifice au fantôme d'un mort, aucune réciprocité n'est due" (Breton 2002b : 215). Il parle aussi de paiements matrimoniaux pour les compensations de mariage qui, pour lui, ne relèvent pas d'une logique de l'équivalence, mais de l'échange identique. 
Cela dit, l'ensemble des contre-arguments à la critique de Stéphane Breton qu'on vient d'avancer ne font qu'atténuer sa portée en montrant que ses bases empiriques ne semblent pas aussi solides qu'elles le paraissent a priori. Les réponses précédentes ne pénètrent pas jusqu'au cœur de sa critique, lequel renvoie moins à un problème de faits que de méthode pour les saisir, voire même d'épistémologie. En effet, l'ultime position de Stéphane Breton, qui résume bien le fond de son argument, est la suivante:

"La monnaie est l'instrument de paiement des dettes, cela va de soi. Dans certains cas, le systèmes des dettes est en quelque sorte authentifié par l'idée générale d'une Dette des agents à l'égard de la puissance sociale. C'est le cas de l'Inde et, grosso modo, de la monnaie occidentale moderne [...]. Dans d'autres cas, [...] [comme] la Mélanésie, la monnaie est l'expression d'une subordination, d'une dépendance des agents à l'égard du Tout social, mais pas nécessairement sous la forme explicite de la dette. Dans ce cas, la monnaie est plutôt, sur le plan de l'échange en général (mais pas forcément marchand) qui est en Mélanésie le niveau d'inscription et de réalisation des valeurs, une sorte de signe de la subordination des niveaux (par exemple des vivants aux ancêtres)" (correspondance personnelle).

Dans cette formulation, on n'est plus renvoyé à l'idéologie particulière des Wodani et on ne peut donc plus répondre, comme on l'a fait plus haut, en tentant de voir dans cette absence de dette l'effet d'une forme de fétichisme. Stéphane Breton affirme simplement qu'il n'y a pas de dette si celle-ci n'apparait pas sous une forme explicite. C'est une position qui se tient et à laquelle on peut adhérer, mais à la condition de distinguer la dette, comme concept subjectif et moral, appartenant au vécu des sujets, de la dette (de vie) comme concept objectif, appartenant d'abord à la pensée logiquement construite des observateurs scientifiques. Ainsi, peut-on tenir ensemble "vérité officielle" et "vérité objective", pour reprendre Pierre Bourdieu. Mais cela appelle une mise au point, voire même une autocritique de la manière ambivalente dont La Monnaie souveraine a utilisé l'idée de dette primordiale en se référant aux seules situations où, effectivement, la dette de vie est bien pensée comme telle (notamment l'Inde, l'Europe chrétienne, etc.) et en la posant simultanément comme (quasi) universelle. Les critiques y ont vu une adhésion à l'idée d'une Dette de vie entre humains et transcendance (et/ou ce qui en tient lieu sur terre) qui, non seulement serait un concept objectif, abstrait, pour saisir un lien social vertical se traduisant par des paiements monétaires unilatéraux, mais aussi serait saisie, pensée comme telle en tout contexte par les humains eux-mêmes. Sans doute, du fait que la thèse a été émise dans un contexte sociétal dans lequel perdure une tradition de penser la dette sur un mode moral, la confusion était-elle largement inévitable. On doit néanmoins en sortir en distinguant clairement la notion de dette, subjective, normative, fait social, de celle de "dette de vie », le véritable concept objectif, abstrait, proposé comme analyseur dans La Monnaie souveraine, fait de pensée destiné à modéliser le lien de «dépendance des agents à l'égard du Tout social ", quel que soit le sens ascendant ou descendant de ce lien et y compris dans les contextes mélanésiens ou autres où cette dette de vie, objective, n'est pas vécue subjectivement comme dette par les agents. 
Il y a objectivement dette verticale de vie chez les Wodani puisque la société réitère le don inaugural de capital-vie que constitue le corps de l'ancêtre primordial, corps démembré, recomposé et transmis sans cesse, « substance paternelle » produite par l'auto-sacrifice de l'ancêtre mythique, «substance» représentée et transformée en «substance sociale" par la monnaie (Breton 2002b: 198), substance du tout social donc, dont la monnaie est la représentation. Ainsi :

«[...] on observe une transmission de monnaie calquée sur le modèle de la transmission de substance masculine [...] qu'elle signifie, qu'elle répète sur le plan symbolique [...]. La monnaie est un sceau authentificatoire [...]. [Il] y a un don premier, celui de l'ancêtre, et il est seulement répété. Cette répétition est la reproduction même de la société" (correspondance personnelle).

Ce don inaugural fonde simultanément la société et son système monétaire qui en est ainsi indissociable ; le capital-vie transmis entre générations, et dont la circulation ininterrompue assure la reproduction de la société, a une expression directement monétaire. Les paiements comblent les déficits corporatif et social de capital-vie en les compensant symboliquement, la monnaie étant tout autant métonymiquement que métaphoriquement la «substance paternelle» elle-même.

La monnaie est de la sorte, chez les Wodani comme chez les 'Aré'aré (de Coppet 1998), en position de souveraineté : elle est immortelle - sa durée de vie excède la succession des générations - et vient d'ailleurs - elle est faite de coquillages dans une société qui ne connaît pas la mer ; elle est médiation stable entre les humains et les ancêtres qui lui conferent son autorité et son pouvoir; elle peut mettre à mort toute personne qui veut la conserver pour son propre compte. Cette souveraineté conférée à la monnaie correspond à une objectivation de la souveraineté. Un tel fétichisme explique sans doute le caractère naturel, évident aux yeux des sociétaires, de la «transmission » de la substance sociale ; il empêche que l'idée d'aliénation à l'égard de cette transmission fasse sens. Car la monnaie, qui représente en ce cas directement la dette verticale de vie, qui est cette dette verticale, n'est pas elle-même pensée comme dette (ce qui est d'ailleurs également le cas dans les sociétés modernes où seule la théorie sociologique la pense comme dette, créance sur le tout social dont le débiteur n'est pas précisé, selon l'expression de Georg Simmel, ce qui signifie qu'elle n'est pas elle-même a priori remboursable).

Ainsi l'inexistence d'une dette verticale, le fait que la transmission du capital de vie ne soit pas vécue comme dette de vie à l'égard des ancêtres serait à corréler avec la souveraineté de la monnaie, avec le fait que la monnaie est souveraine. Cela expliquerait également qu'il n'y pas de problème de confiance dans la monnaie chez les Wodani, tant que rien ne menace la souveraineté de cette dernière, tant que le régime de monnayage reste parfaitement cohérent. Que la monnaie soit en position de souveraineté fait également que la dette de vie, y compris la verticale, est commensurable, le don initiateur de la dette étant sans cesse intégralement répété symboliquement dans la circulation monétaire associée à la circulation des épouses (et plus secondairement des morts par meurtre) entre clans. 
Ainsi la dette diagonale peut-elle dominer la dette verticale au point que celle-ci soit occultée par celle-là dans la reproduction au jour le jour de la société37.

Mais la dette de vie verticale n'en est pas moins toujours en surplomb. On a vu en effet que le concept de dette de vie ne valait pas seulement diagonalement au niveau des clans au sein desquels la vie est transférée et donnée - dette de vie diagonale puisque la verticalité de la filiation est conditionnée par l'alliance horizontale, dès lors qu'il y a tabou de l'inceste -, mais aussi verticalement à l'égard de l'ensemble de la société où les ancêtres et autres esprits donnent la mort - ne donnent la vie que dans la mesure où ils se privent de donner la mort (par maladie) moyennant des sacrifices. Or la mort, autorité souveraine par excellence, est la condition de la vie, ainsi que l'indique clairement le mythe de l'autosacrifice de l'ancêtre primordial fondateur de la société.

Centre national de la recherche scientifique IRISSO, Université Paris Dauphine, Paris theret@dauphine.fr

MOTS CLÉS/KEYWORDS : dette horizontale/ horizontal debt-dette verticale/vertical debt-dette diagonale/ diagonal debt - monnaie exotique/primitive money - monnaie moderne/modern money - don/gift - capital de viel life stock.

37. Alors qu'elle est a contrario mise en avant et en scène dans les sociétés à grande religion universaliste, et non pas inventée par elles comme le suggère Alain Caillé. 
Absi, Pascale

2008 «La part du diable. Métal et monnaie dans les mines du Potosi ", in Evelyne Bauman et al., eds, Argent des anthropologues, monnaie des économistes. Paris, L'Harmattan : 97-114.

Akin, Joel \& David Robbins, eds 1999 Money and Modernity. State and Local Currencies in Melanesia. Pittsburg, University of Pittsburg.

Blanc, Jérôme

2007 Exclusions et liens financiers. Monnaies sociales. Paris, Economica.

Bloch, Maurice \& Jonathan Parry, eds 1989 Money and the Morality of Exchange. Cambridge, Cambridge University Press.

\section{Breton, Stéphane}

2000 "Le monde de la dette", Annales. Histoire, Sciences Sociales 6 : 1361-1366. 2002a «Présentation. Monnaie et économie des personnes ", L'Homme 162 : 13-26. 2002b «Tuer, manger, payer: l'alliance monétaire des Wodani de Papouasie occidentale ", L'Homme 162 : 197-232.

\section{Caillé, Alain}

2000 Anthropologie du don. Le tiers paradigme. Paris, Desclée de Brower. 2002 "Quelle dette de vie?", L'Homme 162 : 243-254.

\section{Carruthers, Bruce \& Sarah Babb}

1996 "The Color of the Money and the Nature of the Value: Greenbacks and Gold in Postbellum America ", American Journal of Sociology 101 (6) : 1556-1591.

\section{Cartelier, Jean}

2007 «The Hypostasis of Money : An Economic Point of View ", Cambridge of Economics 31(2) : 217-233.

\section{Commons, John R.}

1990 [1934] Institutional Economics. Its Place in Political Economy. New BrunswickLondon, Transactions Publishers.

\section{Coppet, Daniel de}

1970 "La monnaie : présence des morts et mesure du temps ", L'Homme 10 (1) : 17-39. 1998 «Une monnaie pour une communauté mélanésienne comparée à la nôtre pour l'individu des sociétés européennes ", in Michel Aglietta \& André Orléan, eds, La Monnaie souveraine... : 159-211.

\section{Détienne, Marcel}

2000 Comparer l'incomparable. Paris, Le Seuil.

Fürer-Haimendorf, Christoph von

1974 «The Sense of Sin in Cross-Cultural Perspective ", Man 9 (4) : 539-556.

\section{Grenier, Jean-Yves}

2000 "Penser la monnaie autrement", Annales. Histoire, Sciences sociales 6: 13351342.

\section{Hénaff, Marcel}

2002 Le Prix de la vérité. Le don, l'argent, la philosophie. Paris, Le Seuil.

\section{Hocart, $M$.}

1978 Rois et courtisans. Paris, Le Seuil.

\section{Laum, Bernhard}

1924 Heiliges Geld, Historische Untersuchung über den Sakralen Ursprung des Geldes. Tübingen, J.C.B. Mohr : 158. [Traduit par Alban Bensa : "Présentation de Genèse et nature de la monnaie, de Bernhard Laum ", Genèses 1992, 8 : 60-85.]

\section{Monnerie, Denis}

2002 «Monnaies de Mono-Alu : valeurs, discontinuités et continuités dans les objets et les relations sociales ", L'Homme 162 : 81-106. 


\section{Parry, Jonathan}

1986 "The Gift, the Indian Gift, and the "Indian Gift" ", Man 21(3) : 453-473.

\section{Parry Jonathan \& Maurice Bloch}

1989 "Introduction : Money and the Morality of Exchange ", in Money and the Morality of Exchange... : 1-32.

Parry, Jonathan \& Maurice Bloch, eds 1989 Money and the Morality of Exchange. Cambridge, Cambridge University Press.

Piron, Sylvain

2002 "La dette de Panurge ", L'Homme $162: 255-270$.

\section{Rospabé, Philippe}

1995 La Dette de vie. Aux origines de la monnaie sauvage. Paris, La DécouverteMAUSS.

\section{Sallnow, Mike}

1989 "Precious Metals in the Andean Moral Economy ", in Jonathan Parry \& Maurice Bloch, eds, Money and the Morality of Exchange... : 213-214.

\section{Scubla, Lucien}

1985 Logiques de la réciprocité. Paris, CREA («Cahiers du CreA » 6).

\section{Servet, Jean-Michel}

1981 "Genèse des forems et pratiques monétaires ", Cahiers Monnaie et financement $11: 357-386$.

1999 Une économie sans argent. Paris, Le Seuil.

\section{Théret, Bruno}

1994a «To Have or to Be. On the Problem of the Interaction Between State and Economy and its Solidarist Mode of Regulation ", Economy and Society 23 (1): $1-46$. 1994b « Le salariat comme forme

d'indépendance entre l'État et le marché ", in Bertrand Bellon et al., L'État et le Marché, Paris, Économica : 68-79.

1998 «De la dualité des dettes et de la monnaie dans les sociétés salariales", in Michel Aglietta \& André Orléan, eds, La Monnaie souveraine... : 253-287.

1999 "Vers un socialisme civil ? Lépreuve de la contrainte démocratique de différenciation de la société ", in Bernard Chavance et al., eds, Capitalisme et socialisme en perspective. Evolution et transformations des systèmes économiques.

Paris, La Découverte : 56-65.

2001 «Saisir les faits économiques. La méthode Commons ", Cahiers d'économie politique 40-41 : 79-137.

2003 «Responsabilité et solidarité : une approche en termes de dette", in Colette Bec \& Giovana Procacci, eds, De la responsabilité solidaire. Mutations dans les politiques d'aujourd'hui. Paris, Syllepse : 51-67.

2007 «La monnaie au prisme de ses crises d'hier et d'aujourd'hui ", in B. Théret, ed., La Monnaie dévoilée par ses crises... : I, 17-74.

Théret, Bruno, ed.

2007 La Monnaie dévoilée par ses crises. Paris, Éd. de l'Ehess, 2 vol.

\section{Zelizer,Viviana}

2005 La Signifiacation sociale de l'argent. Paris, Le Seuil («Liber»). 